\title{
Graphene Triangular Ballistic Rectifier: Fabrication and Characterisation
}

\author{
GREGORY AUTON, ${ }^{1,2,4}$ ROSHAN KRISHNA KUMAR, ${ }^{3}$ ERNIE HILL, ${ }^{1}$ \\ and AIMIN SONG ${ }^{2,5}$
}

1.-Manchester Centre for Mesoscience and Nanotechnology, University of Manchester, Manchester M13 9PL, UK. 2.-School of Electrical and Electronic Engineering, University of Manchester, Manchester M13 9PL, UK. 3.-School of Physics and Astronomy, University of Manchester, Manchester M13 9PL, UK. 4.-e-mail: Gregory.Auton@manchester.ac.uk. 5.-e-mail: A.Song@manchester.ac.uk

It has been shown that graphene can demonstrate ballistic transport at room temperature. This opens up a range of practical applications that do not require graphene to have a band gap, which is one of the most significant challenges for its use in the electronics industry. Here, the very latest high mobility graphene $\left(>100,000 \mathrm{~cm}^{2} \mathrm{~V}^{-1} \mathrm{~s}^{-1}\right)$ fabrication techniques will be demonstrated so that one such device, called the triangular ballistic rectifier (TBR), can be characterised. The TBR is a four-terminal device with a triangular anti-dot at their intersection; two sides of the triangle are positioned and angled such that ballistic carriers from the two input electrodes are redirected like billiard balls to one of the two output contacts irrespective of the instantaneous polarity of the input. A responsivity of $2400 \mathrm{mV} \mathrm{mW}^{-1}$ is demonstrated at room temperature from a low-frequency input signal. The ballistic nature of the device is justified and explained in more detail with lowtemperature measurements.

Key words: Graphene, rectifier, ballistic, nano-device

\section{INTRODUCTION}

Graphene has the highest carrier mobility and mean free path of any known material at room temperature ,and since its conception much work has been done to demonstrate this. ${ }^{1-5}$ However, it was only the introduction of boron nitride (BN) as a substrate that allowed graphene to begin to realise its potential. ${ }^{6}$ Its atomically flat surface, lack of dangling bonds and similar crystallographic structure to graphene proved to make it the perfect medium. Further, by encapsulating graphene between $\mathrm{BN}$ flakes using Van Der Waals interactions, the flakes could be stacked up with clean interfaces making superior devices. ${ }^{7}$ However, encapsulating the graphene meant that electrical contacts to the graphene could not any longer be made through the Basel plane; instead, side contacts had to be used. It turned

(Received August 9, 2016; accepted September 2, 2016; published online September 20, 2016) out that these side contacts or one-dimensional contacts were superior to traditional two-dimensional (2D) contacts. ${ }^{7}$ These advances have allowed devices to be reliably fabricated with carrier mobilities over $100,000 \mathrm{~cm}^{2} \mathrm{~V}^{-1} \mathrm{~s}^{-1}$ at room temperature; this corresponds to a mean free path of over $1 \mu \mathrm{m}$. This incredible property allows the carriers in any device with characteristic dimensions smaller than $1 \mu \mathrm{m}$ to move in the ballistic regime of electron transport. Ballistic electrons behave like billiard balls because there are very few impurities or phonons to scatter off; instead, carriers will scatter off the edges of the graphene.

The ballistic rectifier (BR) is a four-terminal device that utilises this ballistic regime of electron transport, artificial inhomogeneities redirect carriers from either of the two input contacts to one of the two output contacts. The effect is that carriers injected by an alternating current end up at the same output contact irrespective of the instantaneous polarity of the input current, rectifying an 
alternating current (AC) signal into a direct current (DC) signal. The BR do not rely on depletion regions or barrier structures along the direction of electron movement like traditional rectifiers, allowing the carriers to travel without having to overcome a built-in field and therefore have a zero threshold voltage. It is particularly favourable for high-frequency devices to have a low threshold since often the input signal will be very small. The BR also lends itself to high-frequency applications because it has a low parasitic capacitance due to its planar nature. Some of the most promising applications for this technology come from its combination with an antenna, known as a rectenna. The rectenna can be used for a variety of applications in biomedical diagnostics, security imaging, optical heterodyne detection, thermo-electric energy harvesting and cooling. ${ }^{8-10}$

Previous work demonstrated a graphene BR that utilised high field gradients through the two narrow injection leads to redirect the carriers. The result was a device with responsivity as high as $23,000 \mathrm{mV} \mathrm{mW}^{-1}$ and a noise equivalent power (NEP) as low as $0.64 \mathrm{pW} \mathrm{Hz}^{-1 / 2}$ at room temperature. ${ }^{5}$ In this work, we present a device, the triangular ballistic rectifier (TBR), that uses specular scattering at the edge of a triangular anti-dot to redirect the carriers; the TBR has previously been demonstrated in other 2D electron gases (2DEGs). ${ }^{11-13}$ These InGaAs/AlGaAs hetero-structure TBRs demonstrated full-wave rectifying properties similar to a bridge rectifier which consists of four traditional diodes.

\section{FABRICATION METHODS}

The devices that feature in this work were all fabricated using the very latest high-mobility fabrication techniques to achieve the highest mobility possible. In order to find the graphene and judge the thickness of the BN using its colour, all flakes were exfoliated on $290 \mathrm{~nm} \mathrm{SiO}$ thermally grown on highly doped Si. Firstly, the wafers were cleaned using a standard solvent clean and baked on a hotplate while the tape peals of $\mathrm{BN}$ and graphite crystals were prepared. Each peal was then pressed onto a wafer and left for $1 \mathrm{~h}$ to increase the yield of flakes. Meanwhile, a clean polymethyl methacrylate (PMMA) membrane was prepared by spinning and baking a sacrificial layer of PVA and then a $400-\mathrm{nm}$ film of PMMA on a clean $\mathrm{Si}$ wafer. A 3-mm circle was scratched through both layers of the resist and water pipetted into the gouge to dissolve the PVA, then once the PMMA membrane was free from the Si it was floated in a beaker of water. A 2-mm washer could then be used to fish the membrane from the water so that it was suspended and then dried gently over a hotplate.

The tape was pealed at a low angle slowly from the $\mathrm{SiO}_{2}$, the resulting wafers being observed in an optical microscope. The optical contrast method for finding flakes (which has been discussed previously $^{14,15}$ ) was used to find two $\sim 30$-nm BN flakes and a mono-layer graphene flake. Figure $1 \mathrm{a}$ is a
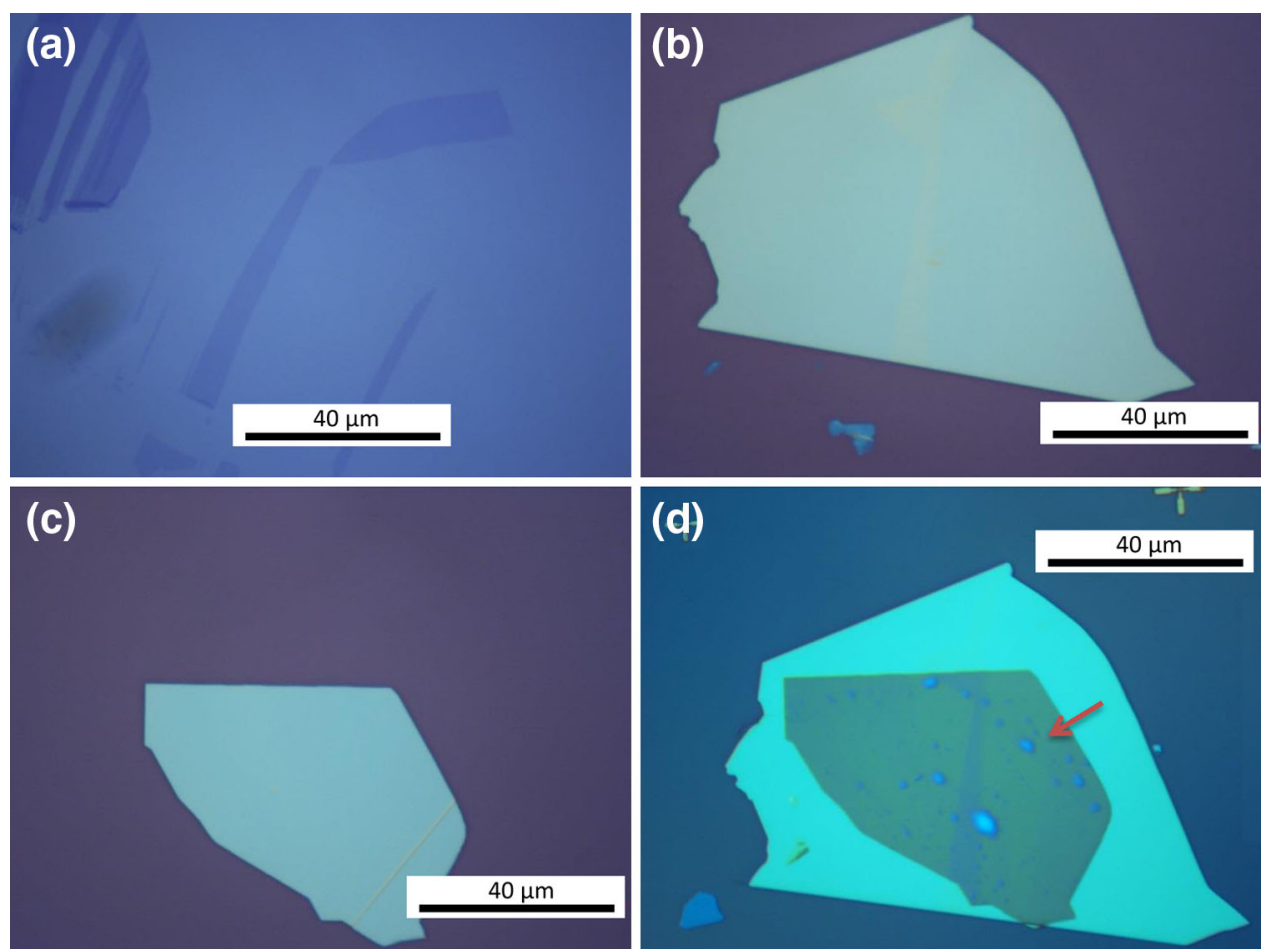

Fig. 1. (a-c) Optical micrographs at $\times 100$ magnification of the graphene flake, the bottom layer of boron nitride $(B N)$ and the top layer of $B N$, respectively. (d) Differential Interference contrast optical micrograph at $\times 100$ magnification of the final stack of flakes, the arrow indicates where the graphene was. 
$\times 100$ optical micrograph showing the monolayer flake on $290 \mathrm{~nm} \mathrm{SiO}_{2}$ that was used to make the device demonstrated here. In Fig. $1 b$ and $c$ are $\times 100$ optical micrographs showing the bottom and top layer BN flakes, respectively, used for this device. In order to pick up the top layer of $\mathrm{BN}$ using the membrane, the washer was mounted onto an arm connected to a micro-manipulator. The suspended membrane was then lowered over the $\mathrm{BN}$ in a microscope until it was in contact with the surface, the substrate being held down with a vacuum so that the membrane could be removed from the surface, picking up the flake. Then, in turn, the graphene flake and the bottom layer of BN could be picked up and finally placed on a target substrate. Figure $1 d$ shows a $\times 100$ optical micrograph of the final structure of stacked flakes, in which differential interference contrast has been used to increase the contrast of the graphene flake indicated by the arrow.

Contacts were then defined using standard bi-layer PMMA electron beam (ebeam) lithography techniques and the whole flake stack was etched through using an Oxford Plasma Lab in order to leave the edge of the graphene exposed. The etch parameters were as follows: flow rates of $17.5 \mathrm{ccm}$ of $\mathrm{CHF}_{3}$ and $8 \mathrm{ccm}$ of $\mathrm{O}_{2}$ were used with RF power $5 \mathrm{~W}$ and ICP $50 \mathrm{~W}$ at a pressure of $1.33 \mathrm{~Pa}$. This procedure etched the graphene and BN without damaging the PMMA so that the same PMMA could be used to lift-off the 3/ $80 \mathrm{~nm} \mathrm{Cr} / \mathrm{Au}$ contacts. By using the same PMMA to expose the graphene edge to make contact and pattern the contacts, a polymer/solvent-free interface could be achieved, lowering their resistance and increasing their reliability. An etch mask was then defined using single-layer PMMA and ebeam lithography so that the geometry of the device could be defined with the same etch procedure.

Figure $2 \mathrm{a}$ and $\mathrm{b}$ shows optical micrographs at $\times 5$ and $\times 100$, respectively, of the finished graphene device, Hall bar and gold contacts, and the location of the TBR. Figure 2c shows an atomic-force micrograph of the active region of the TBR showing the geometry of the device with its parts labelled. Typical specular carrier trajectories, demonstrating the asymmetric nature of the device, are indicated. The widths of channels $\mathrm{S}$ and $\mathrm{D}$ were measured as $177 \mathrm{~nm}$ and $248 \mathrm{~nm}$, respectively.

\section{RESULTS AND DISCUSSION}

To demonstrate that these devices operate in the ballistic regime, it is important that the mean free path was longer than the path length through the device. A standard Hall bar geometry was included in the design next to the TBR so that transport
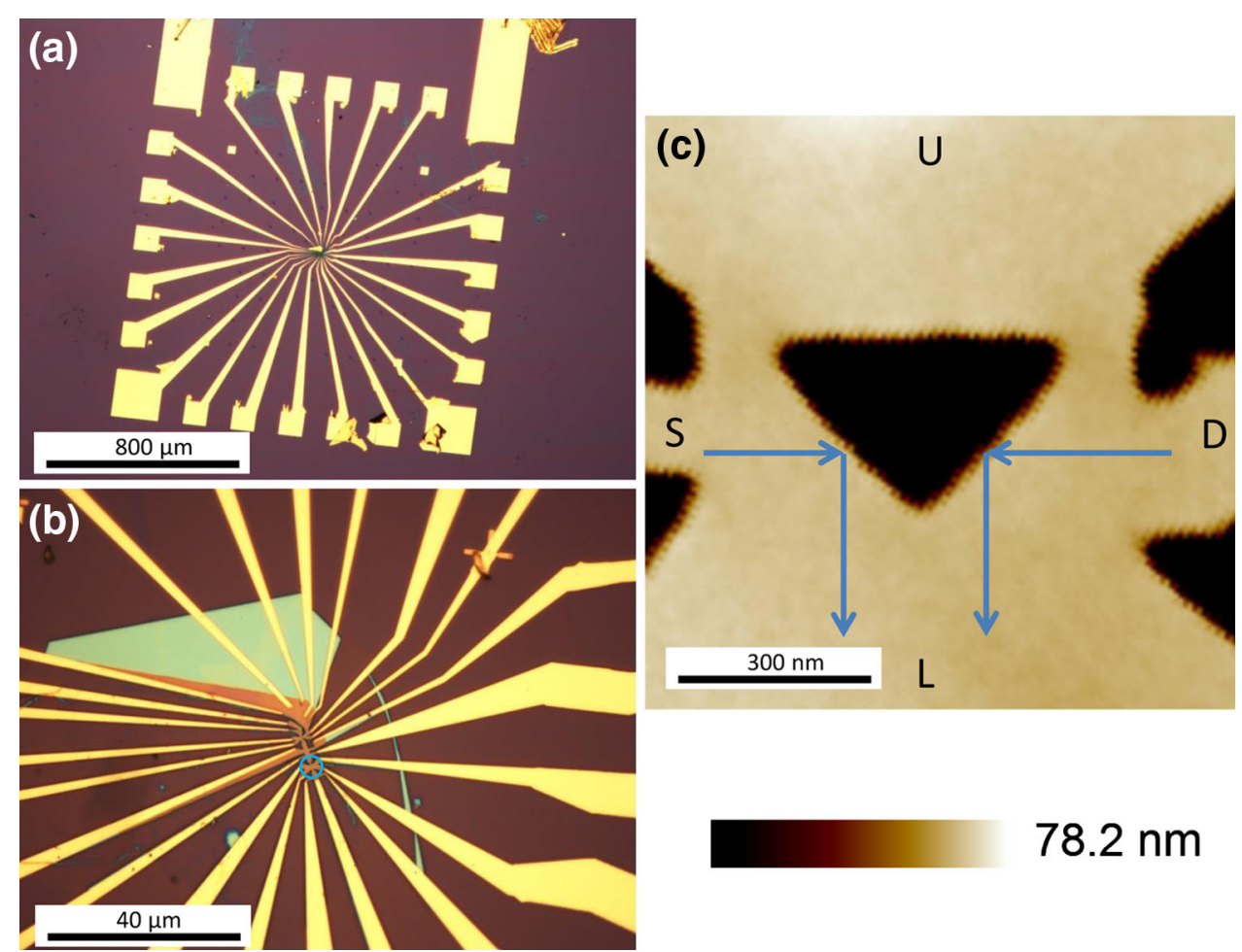

Fig. 2. (a, b) Optical micrographs at $\times 5$ and $\times 100$, respectively, of the finished graphene device, Hall bar and gold contacts, the circle indicates the location of the triangular ballistic rectifier (TBR). (c) Atomic-force micrograph of the active region of the TBR showing the geometry of the device. The letters $S, D, U$ and $L$ label the source, drain, upper and lower contacts. The arrows indicate typical carrier trajectories, demonstrating the asymmetric nature of the device specularly reflects the carriers to $L$ rather than $U$ irrespective of the input polarity. 
(a)

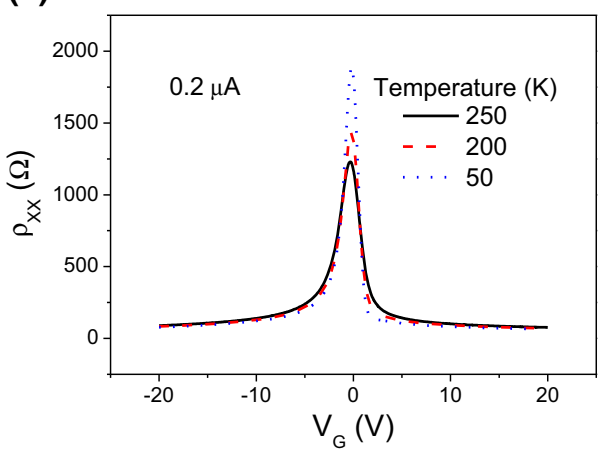

(b)

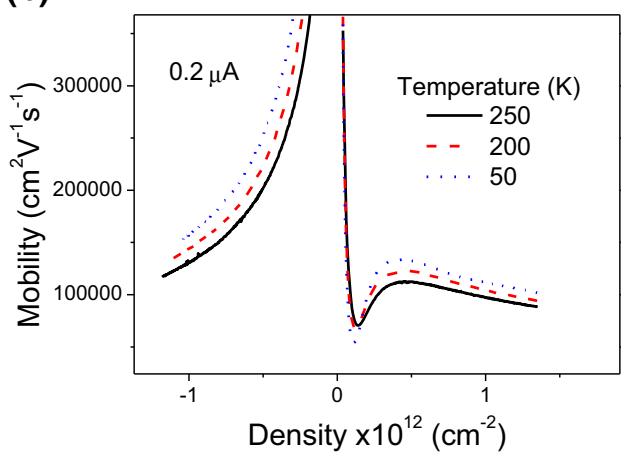

Fig. 3. (a) The dependency of resistivity on gate voltage $\left(V_{G}\right)$ measured along the Hall bar region of the device with a four-point setup while $0.2 \mu \mathrm{A}$ was applied at 250,200 and $50 \mathrm{~K}$. (b) The dependency of carrier mobility $(\mu)$ on carrier density derived from Hall and resistivity measurements while $0.2 \mu \mathrm{A}$ was applied at 250, 200 and $50 \mathrm{~K}$.

measurements can discover out the properties of the graphene itself.

Figure 3a shows longitudinal resistivity $\left(\rho_{X X}\right)$ against gate voltage $\left(V_{\mathrm{G}}\right)$ while a constant current of $0.2 \mu \mathrm{A}$ is applied at different temperatures. The neutrality point of the device is at $V_{\mathrm{G}}=1 \mathrm{~V}$ at room temperature showing the high quality of the device due to the lack of doping. Figure $3 \mathrm{~b}$ shows mobility $(\mu)$ as a function of carrier density. Mobility and density are measured by Hall and $\rho_{X X}$ measurements. Since $l_{e}=(\hbar / e) \mu(\pi n)^{1 / 216}$ a mobility of $100,000 \mathrm{~cm}^{2} \mathrm{~V}^{-1} \mathrm{~s}^{-1}$ and density of $10^{12}$ corresponds to a mean free path of $1.17 \mu \mathrm{m}$ and the path length through the device is $\sim 1 \mu \mathrm{m}$, this device is in the ballistic regime of electron transport. The width and length of the Hall bar are $1 \mu \mathrm{m}$ making it hard to claim that any measurements of the $l_{e}$ above this value are valid; instead, $1 \mu \mathrm{m}$ is only a lower bound for $l_{e}$ in this device.

Figure 4a shows the voltage between $L$ and $U$ $\left(V_{\mathrm{LU}}\right)$ as a function of the current between $S$ and $D$ $\left(I_{\mathrm{SD}}\right)$ for different $V_{\mathrm{G}}$. One important feature of the TBR is that the output is carrier-type-dependent, both carrier types will bounce to $L$ and so when the majority carriers are electrons (holes), the voltage between $L$ and $U$ will be negative (positive). Graphene is an ambipolar material so it should be possible to see both types of output. The figure clearly shows both positive curvature and negative curvature corresponding to carrier majorities of holes and electrons respectively. The curves are parabolic in nature, as it is predicted by the Büttiker-Landauer formalism that the output should be quadratic. ${ }^{17}$ This can be explained by remembering that fabrication procedures are not perfect and any small misalignment between $L$ and $U$ vertically will contribute to a linear output. Removing this linear term can be achieved by adding the data to its reverse and dividing by two to give the asymmetry, defined as Asymmetry $=$ $\left(V_{\mathrm{LU}}\left(I_{\mathrm{SD}}\right)+V_{\mathrm{LU}}\left(-I_{\mathrm{SD}}\right)\right) / 2$. Figure $4 \mathrm{~b}$ shows the asymmetry of $V_{\mathrm{LU}}$ as a function of $I_{\mathrm{SD}}$ for different
$V_{\mathrm{G}}$, the quadratic response of the device is evident here. It is important to note that any linear contribution would cancel out in a high frequency devices and would not contribute to the output.

Figure $5 \mathrm{a}$ shows $V_{\mathrm{LU}}$ as a function of $V_{\mathrm{G}}$ while different $190 \mathrm{~Hz}$ AC $I_{\mathrm{SD}}$ are applied at room temperature. This experiment tests the low frequency ac response of the device as the Fermi level is changed in the device. Firstly, despite the proximity to the Hall bar used to measure the transport properties of the graphene, the neutrality point for this device is at $-5 \mathrm{~V}$. This discrepancy could be due to self-doping of the narrow channels by defects at the edge ${ }^{18,19}$ or it could be due to high current densities through the narrow channels creating electrical stress. It could even be due to a localised hydrocarbon contamination trapped between the $\mathrm{BN}$ and the graphene; however, this would normally be visible on Fig. $2 \mathrm{c}$ and it is not.

In order to understand the variation with $V_{G}$ the Büttiker-Landauer formalism should be considered from $^{17}$ :

$$
V_{\mathrm{LU}} \approx-\frac{h}{e^{2}} \frac{3 \hbar}{4 e E_{\mathrm{F}} N_{\mathrm{SD}}} \frac{\sin 2 \vartheta_{0} I_{\mathrm{SD}}^{2}}{2 N_{\mathrm{LU}}-3 N_{\mathrm{SD}}\left(1-\sin \vartheta_{0}\right)^{2}}
$$

where $E_{\mathrm{F}}$ is the Fermi energy, $V_{\mathrm{LU}}$ is the voltage between the lower and upper contacts, $N_{\mathrm{SD}}$ is the number of propagating modes between the source and the drain, $N_{\mathrm{LU}}$ is the number of propagating modes between the lower and upper contacts and $\vartheta_{0}$ is the angle between the normal of the narrow channel and the point that it will just miss the triangle and go into the upper electrode. The number of propagating modes in any narrow channel $\left(N_{X}\right)$ will change with $N_{X}=\frac{2 W_{X}}{\lambda_{\mathrm{F}}}$ where $\lambda_{\mathrm{F}}$ is the Fermi wavelength and $W_{X}$ is the physical width of the channel. ${ }^{5,20,21}$

The overall result of Eq. 1 is that there is an increase in output as the Dirac point is approached because the Fermi energy decreases and the Fermi wavelength of the carriers increases, and therefore 
(a)

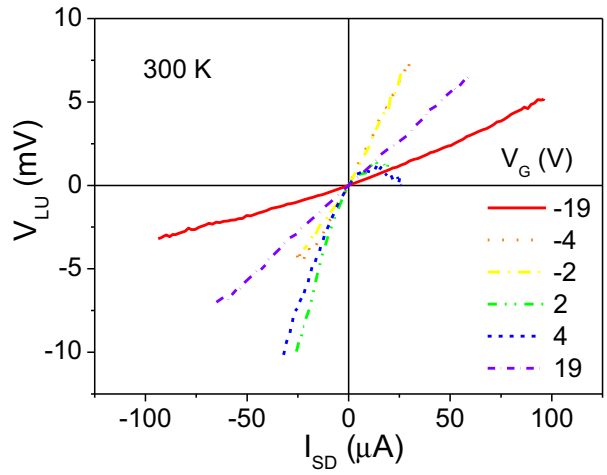

(b)

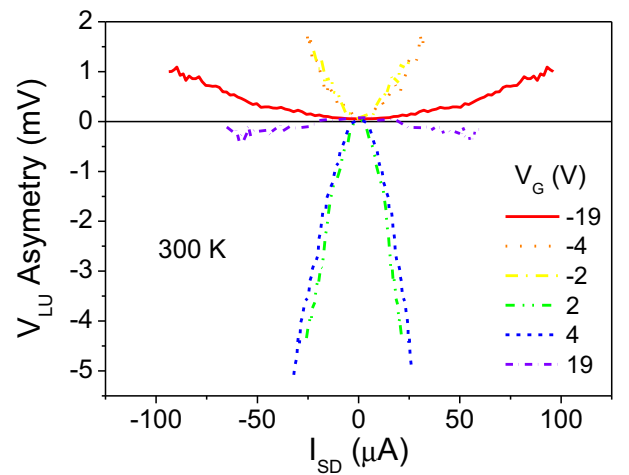

Fig. 4. (a) The voltage between $L$ and $U\left(V_{L U}\right)$ as a function of the current between $S$ and $D\left(I_{S D}\right)$ for different $V_{G}$ at room temperature for a TBR. (b) The asymmetry of $V_{\mathrm{LU}}$ as a function of $I_{\mathrm{SD}}$ for different $V_{\mathrm{G}}$, where $V_{\mathrm{LU}}$ Asymmetry $=\left(V_{\mathrm{LU}}\left(I_{\mathrm{SD}}\right)+V_{\mathrm{LU}}\left(-I_{\mathrm{SD}}\right)\right) / 2$.

(a)

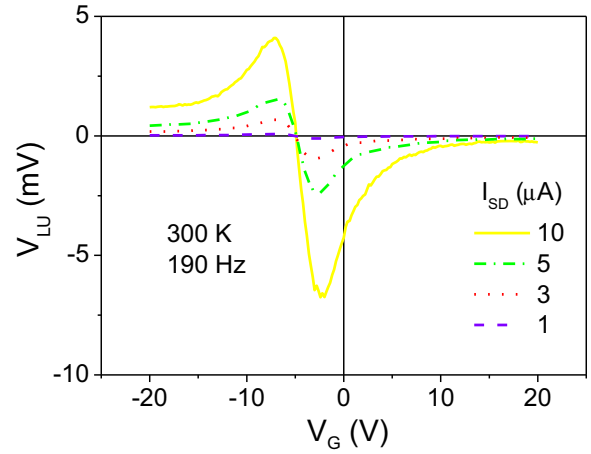

(c)

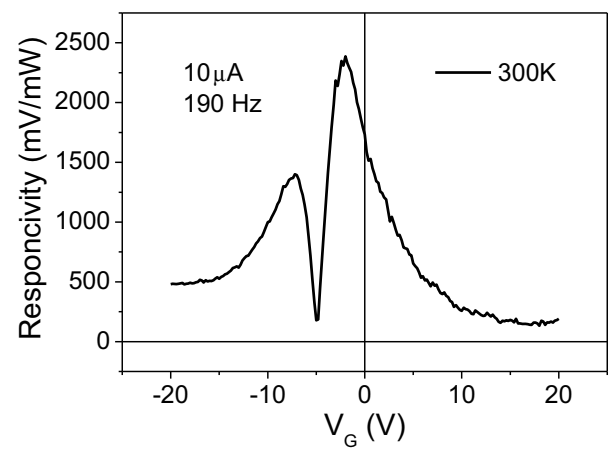

(b)

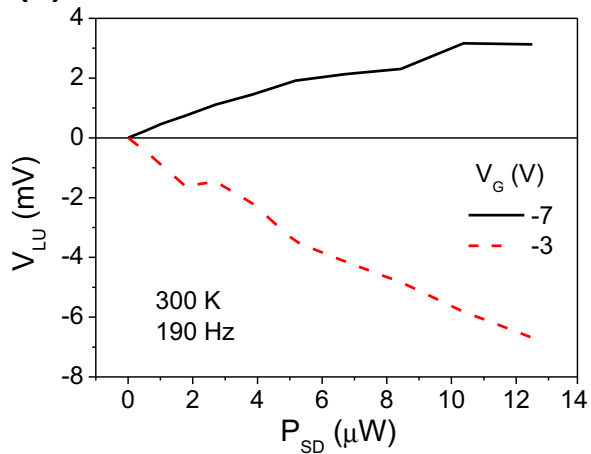

(d)

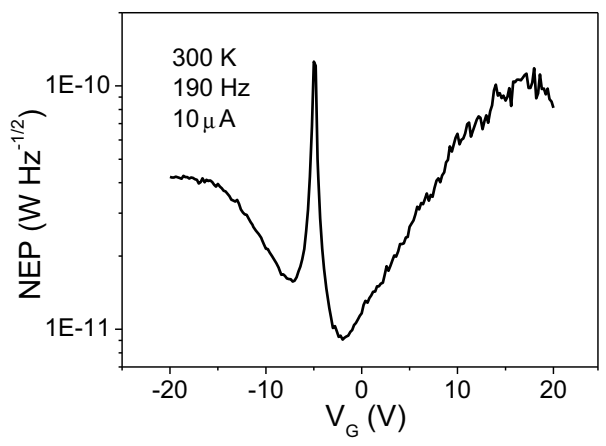

Fig. 5. (a) $V_{L U}$ as a function of $V_{G}$ while different $190 \mathrm{~Hz} A C I_{S D}$ are applied at room temperature. (b) Dependence of $V_{L U}$ on ac power input $\left(P_{S D}\right)$ at room temperature for hole-type carriers $\left(V_{G}=-7 \mathrm{~V}\right)$ and for electron-type carriers $\left(V_{G}=-3 \mathrm{~V}\right)$. (c) Responsivity as a function of $V_{G}$ measured from (a) at room temperature. (d) noise equivalent power (NEP) as a function of $V_{\mathrm{G}}$ derived from (c) and $R_{\mathrm{SD}}$.

there is a reduction in the number of lateral conduction modes. However, due to thermal excitation and local inhomogeneities close to the Dirac point, there is coexistence of both carrier types which leads to a linear change in output.

Figure $5 \mathrm{~b}$ shows the dependence of $V_{\mathrm{LU}}$ on $\mathrm{AC}$ power input $\left(P_{\mathrm{SD}}\right)$ at room temperature for holetype carriers $\left(V_{\mathrm{G}}=-7 \mathrm{~V}\right)$ and for electron-type carriers $\left(V_{G}=-3 \mathrm{~V}\right)$. Since the device is expected to be quadratic as the voltage is increased, it is also expected to be linear as power is increased if it is
Ohmic. Here, we can see that it is linear at low power inputs but then starts to plateau, this could be due to current density going as $J \propto E^{\alpha}$ at high field differentials where $1>\alpha>1.5$ and $E$ is the electric field. ${ }^{22,23}$ Figure $5 \mathrm{c}$ and $\mathrm{d}$ shows responsivity and NEP against $V_{\mathrm{G}}$ where responsivity was measured from DC $V_{\mathrm{LU}}$ divided by AC $P_{\mathrm{SD}}$ and NEP was calculated from the theoretical thermal noise, $\sqrt{4 k T R_{\mathrm{LU}}}$ divided by the responsivity. The peak responsivity measured was $1401 \mathrm{mV} \mathrm{mW}^{-1}$ for holes and $2387 \mathrm{mV} \mathrm{mW}^{-1}$ for electrons, 
(a)

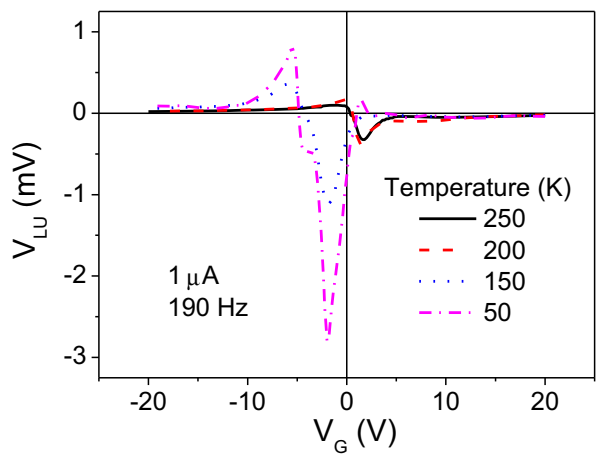

(b)

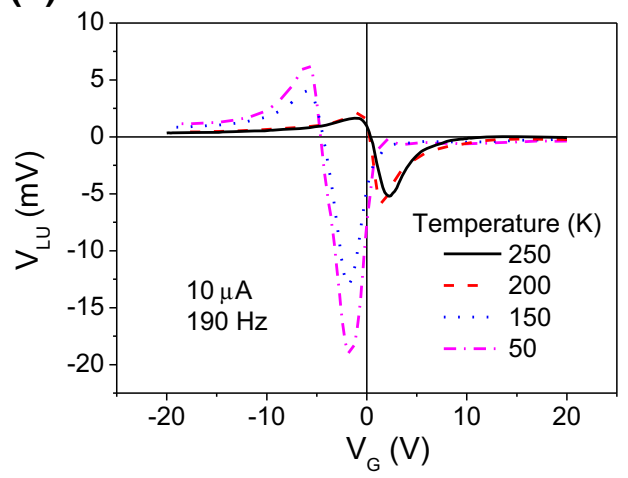

Fig. 6. (a, b) $V_{\mathrm{LU}}$ as a function of $V_{\mathrm{G}}$ while a constant $1 \mu \mathrm{A}$ and $10 \mu \mathrm{A}$, respectively, $190 \mathrm{~Hz} \mathrm{AC} I_{\mathrm{SD}}$ is applied at different temperatures.

corresponding to minima in NEP of $15.7 \mathrm{pW} \mathrm{Hz}^{-1 / 2}$ for holes and $9.1 \mathrm{pW} \mathrm{Hz}^{-1 / 2}$ for electrons.

Figure $6 \mathrm{a}$ and $\mathrm{b}$ shows $V_{\mathrm{LU}}$ as a function of $V_{\mathrm{G}}$ while a constant $1 \mu \mathrm{A}$ and $10 \mu \mathrm{A}$, respectively, $190 \mathrm{~Hz}$ AC $I_{\mathrm{SD}}$ is applied at different temperatures. These graphs clearly demonstrate the ballistic nature of the devices, as lattice vibrations are reduced at low temperature, and the mean free path of the graphene increases resulting in better device performance. As previously discussed in Ref. 5, any contribution from thermal rectification would reduce at low temperature and would in fact be the opposite polarity to the ballistic effect. However, there is a clear discrepancy in the data where the Dirac point has moved for the two higher temperatures so that it is now nearly intrinsically doped. This could be the result of prolonged high current density through the device causing current annealing (the lowest temperature measurements were made first).

Oscillations are present in Fig. 5a, and these are the result of lateral quantum confinement predicted in the Büttiker-Landauer formalism. In Eq. 1, these are represented in the changing number of modes that can propagate through the narrow channels as $V_{\mathrm{G}}$ is changed. The phenomenon is well described in Ref. 5 but the channel widths $S$ and $D$ in this work were less uniform leading to a much less pronounced effect. The oscillations actually wash out at higher input currents because the carrier momentum distribution is more defuse due to current heating of the carriers which is why they are not obvious in Fig. 5b.

\section{CONCLUSIONS}

In conclusion, a four-terminal triangle ballistic rectifier has been fabricated with an extremely high mobility. The ballistic properties of the graphene have been verified and the working principle of the device has been checked. Lateral quantum confinement modes have been observed in the output of the device at low temperatures The resulting device has a peak responsivity of $1401 \mathrm{mV} \mathrm{mW}^{-1}$ for holes and
$2387 \mathrm{mV} \mathrm{mW}^{-1}$ for electrons, with a corresponding minimum NEP of $15.7 \mathrm{pW} \mathrm{Hz}^{-1 / 2}$ for holes and $9.1 \mathrm{pW} \mathrm{Hz}^{-1 / 2}$ for electrons.

\section{ACKNOWLEDGEMENTS}

This work has been supported by the Engineering and Physical Sciences Research Council (Grant Nos. EP/M507969/1, EP/G03737X/1).

\section{OPEN ACCESS}

This article is distributed under the terms of the Creative Commons Attribution 4.0 International License (http://creativecommons.org/licenses/by/4.0/), which permits unrestricted use, distribution, and reproduction in any medium, provided you give appropriate credit to the original author(s) and the source, provide a link to the Creative Commons license, and indicate if changes were made.

\section{REFERENCES}

1. X. Du, I. Skachko, A. Barker, and E.Y. Andrei, Nat. Nanotechnol. 3, 491 (2008).

2. A.S. Mayorov, R.V. Gorbachev, S.V. Morozov, L. Britnell, R. Jalil, L.A. Ponomarenko, P. Blake, K.S. Novoselov, K. Watanabe, T. Taniguchi, and A.K. Geim, Nano Lett. 11, 2396 (2011)

3. S. Masubuchi, K. Iguchi, T. Yamaguchi, M. Onuki, M. Arai, K. Watanabe, T. Taniguchi, and T. Machida, Phys. Rev. Lett. 109, 036601 (2012).

4. A.K. Singh, G. Auton, E. Hill, and A. Song, Carbon 84, 124 (2015).

5. G. Auton, J. Zhang, R.K. Kumar, H. Wang, X. Zhang, Q. Wang, E. Hill, and A. Song, Nat. Commun. 7, 11670 (2016).

6. C. Dean, A. Young, I. Meric, C. Lee, L. Wang, S. Sorgenfrei, K. Watanabe, T. Taniguchi, P. Kim, and K. Shepard, Nat. Nanotechnol. 5, 722 (2010).

7. L. Wang, I. Meric, P.Y. Huang, Q. Gao, Y. Gao, H. Tran, T. Taniguchi, K. Watanabe, L.M. Campos, D.A. Muller, J. Guo, P. Kim, J. Hone, K.L. Shepard, and C.R. Dean, Science 342,614 (2013).

8. M.N. Gadalla and A. Shamim, in Microwave Conference (EuMC), 2014 44th European (2014)

9. M.N. Gadalla, M. Abdel-Rahman and A. Shamim, Sci. Rep. $4,4270(2014)$

10. J.O. McSpadden, L. Fan, and K. Chang, IEEE Trans. Microw. Theory Technol. 46, 2053 (1998). 
11. A.M. Song, P. Omling, L. Samuelson, W. Seifert, I. Shorubalko, and H. Zirath, Jpn. J. Appl. Phys. 40, L909 (2001).

12. A. Löfgren, I. Shorubalko, P. Omling, and A. Song, Phys. Rev. B 67, 195309 (2003).

13. A.M. Song, A. Lorke, A. Kriele, J.P. Kotthaus, W. Wegscheider, and M. Bichler, Phys. Rev. Lett. 80, 3831 (1998).

14. P. Blake, E.W. Hill, A.H. Castro Neto, K.S. Novoselov, D. Jiang, R. Yang, T.J. Booth, and A.K. Geim, Appl. Phys. Lett. 91, 063124 (2007).

15. K.S. Novoselov, A.K. Geim, S.V. Morozov, D. Jiang, Y. Zhang, S.V. Dubonos, I.V. Grigorieva, and A.A. Firsov, Science 306, 666 (2004).
16. A.H. Castro Neto, N.M.R. Peres, K.S. Novoselov, and A.K. Geim, Rev. Mod. Phys. 81, 109 (2009).

17. A.M. Song, Phys. Rev. B 59, 9806 (1999).

18. K. Nakada, M. Fujita, G. Dresselhaus, and M.S. Dresselhaus, Phys. Rev. B 54, 17954 (1996).

19. M. Ridene, J.C. Girard, L. Travers, C. David, and A. Ouerghi, Surf. Sci. 606, 1289 (2012).

20. M. Büttiker, Y. Imry, R. Landauer, and S. Pinhas, Phys. Rev. B 31, 6207 (1985).

21. R. Landauer and M. Büttiker, Phys. Rev. Lett. 54, 2049 (1985).

22. N. Vandecasteele, A. Barreiro, M. Lazzeri, A. Bachtold, and F. Mauri, Phys. Rev. B 82, 045416 (2010).

23. B. Rosenstein, M. Lewkowicz, H.C. Kao, and Y. Korniyenko, Phys. Rev. B 81, 041416 (2010). 